\title{
Prospective Evaluation of Opioid Consumption Following Carpal Tunnel Release Surgery
}

HAND

2017, Vol. 12(I) 39-42

(C) American Association for Hand Surgery 2016

DOI: $10.1177 / 15589447 / 6646765$

hand.sagepub.com

\author{
Talia Chapman', Nayoung Kim², Mitchell Maltenfort ${ }^{2}$, and Asif M. Ilyas ${ }^{2}$
}

\begin{abstract}
Background: Postoperative pain management and opioid consumption following carpal tunnel release (CTR) surgery may be influenced by many variables. To understand factors affecting opioid consumption, a prospective study was undertaken with the hypothesis that CTR performed under local anesthesia (wide awake local anesthesia with no tourniquet [WALANT]) would result in increased opioid consumption postoperatively compared with cases performed under sedation. Methods: All patients undergoing open CTR surgery were consecutively enrolled over a 6-month period. Information collected included patient demographics, surgical technique, amount and type of narcotic prescribed, number of pills taken, and type of anesthesia. Results: 277 patients were enrolled ( $56 \%$ women, $44 \%$ men). On average, 2 I pills were prescribed, and 4.3 pills (median $=2$ ) were consumed. There was no difference in consumption between patients who received WALANT (78 cases) versus (198 cases) sedation (4.9 vs 3.9 pills, respectively) $(P=.22)$. There was no difference in opioid consumption based on insurance type $(P=.47)$ or type of narcotic $(P=.85)$. However, more men consumed no opioids $(47 \%)$ compared with women $(36 \%)(P<.05)$ and older patients consumed less than younger patients $(P<.05)$. Conclusions: Opioid consumption following CTR is more influenced by age and gender, and less influenced by anesthesia type, insurance type, or the type of opioid prescribed. Many more opioids were prescribed than needed, on an average of 5:I. Many patients, particularly older patients, do not require any opioid analgesia after CTR.
\end{abstract}

Keywords: carpal tunnel release, opioid consumption, outpatient surgery, narcotic usage, soft tissue procedure

\section{Introduction}

Currently in the United States, there is an unprecedented prescription drug overdose epidemic, and prescription opioids are a large contributor to this problem. In the past 10 years, there has been a steady increase in the number of opioid analgesics prescribed. The estimated number of prescriptions filled for opioids exceeded 256 million in the United States in 2009, with 234 million prescriptions for immediate-release opioids and 22.9 million for extendedrelease opioids. Compared with 2002, this represents a $42 \%$ increase in opioid prescriptions with a $146 \%$ increase in emergency room opioid prescriptions. ${ }^{5,10}$

Similarly, the abuse of opioid narcotics has increased at a frightening pace. In the United States from 2010 to 2011, the source of over half of prescription opioids for nonmedical use was a friend or relative. ${ }^{9}$ Between 1997 and 2007, retail sales of oxycodone rose by $866 \%$, sales of hydromorphone rose by $319 \%$, sales of hydrocodone rose by $280 \%$, and sales of morphine rose by $222 \%{ }^{6}$ From 1999 to 2010, the number of drug-poisoning deaths involving opioids more than quadrupled. ${ }^{4}$ In 2008, opioid analgesics were involved in 14800 drug overdose deaths, compared with 11500 drug overdose deaths in 2007-an increase of over 3000 deaths in 1 year. ${ }^{7}$ From 2004 to 2008, according to the Centers for Disease Control and Prevention, emergency department visits resulting from the nonmedical use of prescription opioids increased by $111 \%{ }^{2}$ This is an exceedingly dangerous trend.

In addition, recent studies have shown that there is potentially an excessive amount of narcotics being prescribed for postoperative pain management. This practice of overprescribing has vast economic and medical ramifications and can often lead to unintentional opioid abuse. ${ }^{1,3}$ One study of 250 patients after outpatient upper extremity surgery reported that all but 5 patients were prescribed an

\footnotetext{
'Thomas Jefferson University Hospital, Philadelphia, PA, USA

${ }^{2}$ The Rothman Institute, Philadelphia, PA, USA

Corresponding Author:

Talia Chapman, Thomas Jefferson University Hospital, 1025 Walnut

Street, Suite 516, Philadelphia, PA 19107, USA.

Email: talia.chapman10@gmail.com
} 
opioid for analgesia. Prescriptions were most commonly for 30 tablets. At total of $77 \%$ of patients used 15 tablets or fewer and 4639 opioid tablets remained unused and available for potential abuse. ${ }^{8}$ It is a physician's responsibility to treat pain adequately following an acute surgical event; however, it may be that we are overprescribing narcotics and further contributing to the growing opioid problem in this country. An accurate understanding of realistic opioid consumption following outpatient surgery is imperative to treat pain appropriately relative to the surgery being performed without leading to excess narcotic prescribing.

To better understand practical opioid consumption following outpatient hand surgery, we prospectively reviewed narcotic consumption by patients undergoing open carpal tunnel release (CTR) surgery, the most common surgery performed by hand surgeons. It was presumed that postoperative pain management and opioid consumption following CTR may be influenced by a number of variables including patient demographics, and type of anesthesia. The study hypothesis was that patients undergoing CTR under monitored anesthesia care (MAC) would require less opioid analgesia postoperatively, regardless of patient demographics, than patients undergoing CTR under wide awake local anesthesia with no tourniquet (WALANT).

\section{Materials and Methods}

After obtaining institutional review board approval, all patients undergoing open CTR by 8 hand fellowshiptrained orthopedic surgeons were prospectively collected over 6 months. Informed consent was obtained from all individual participants included in the study. Inclusion criteria included all patients undergoing primary unilateral CTR via open technique. Exclusion criteria consisted of all revision CTRs, endoscopic releases, or CTR surgery performed with concomitant soft tissue procedures of the hand. Enrolled patients recorded both the number of opioid pain medication pills consumed and the total number of postoperative days the pills were utilized. Additional variables evaluated included age, gender, insurance, procedure type, and anesthesia. Statistical tests used to perform this analysis included unpaired $t$ tests, analysis of variance, and chi-square tests.

\section{Results}

\section{Demographics}

A total of 277 patients meeting the inclusion criteria were enrolled, which included 154 women (56\%) and 123 men (44\%). The average patient age was 64.6 years (range, 22-90 years). Relative to anesthesia type, 78 procedures were performed under local anesthesia (WALANT) whereas 198 procedures were performed under MAC. One case was performed
Table I. Summary of Demographic Data.

\begin{tabular}{ll}
\hline Total & 277 cases \\
Men & $123(44 \%)$ \\
Women & $154(56 \%)$ \\
Average age & $64.6($ range, $22-90)$ \\
Average No. of pills prescribed & 21 (range, $0-42)$ \\
Average No. of pills used & 4.3 (median $=2)$ \\
WALANT & 78 cases \\
Medicare & $44(56.5 \%)$ \\
Private & $32(41 \%)$ \\
Workers' compensation & $2(2.5 \%)$ \\
MAC & 198 cases \\
Medicare & $74(37.5 \%)$ \\
Private & $118(59.5 \%)$ \\
Workers' compensation & $6(3 \%)$ \\
\hline
\end{tabular}

Note. WALANT = wide awake local anesthesia with no tourniquet; MAC $=$ monitored anesthesia care.

under general anesthesia, which was excluded in the analysis (Table 1).

Among the MAC cases, insurance types included 74 with Medicare, 118 with private insurance, and 6 with workers' compensation insurance. Among the WALANT cases, insurance types included 44 with Medicare, 32 with private insurance, and 2 with workers' compensation (Table 1).

All patients received a prescription for an opioid painkiller of either Percocet ${ }^{\mathrm{TM}}$ or oxycodone equivalent, Vicodin $^{\mathrm{TM}}$ or hydrocodone equivalent, or Tylenol 3. On average, 21 pills (range, 0-42) were prescribed to each patient, and on average only 4.3 pills $($ median $=2)$ were consumed among all cases per patient postoperatively (Table 1 ). Only $13 \%$ of patients consumed more than 10 opioids in the entire study cohort.

\section{Analysis of Opioid Consumption Based on Anesthesia Type}

Among all cases performed, there was no statistical difference in the mean number of opioid pills consumed between those who had WALANT versus MAC anesthesia (4.98 vs 3.94 pills, $P=.22$; Figure 1).

\section{Analysis of Opioid Consumption Based on Insurance Type}

Similarly, there was no difference in average opioid consumption when we compared insurance types. Patients with private insurance consumed on average 4.67 pills compared with 3.72 pills for Medicare patients and 3.62 pills for worker's compensation patients $(P=.47$, Figure 2$)$. When patients with private insurance were directly compared with Medicare patients, there was still no statistically 


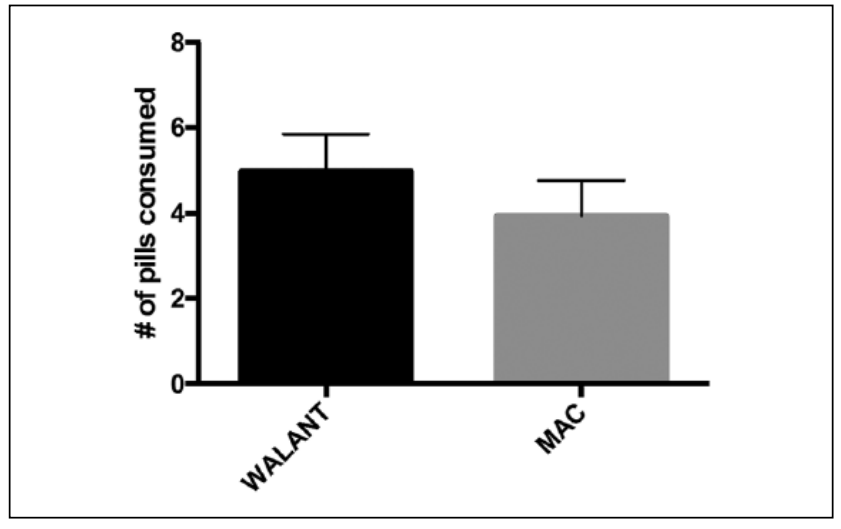

Figure I. Comparing narcotic consumption in open CTR for WALANT versus MAC sedation.

Note. There was no statistical difference in the mean number of opioid pills consumed between those who had WALANT versus MAC anesthesia (4.98 vs 3.94 pills, $P=.22$ ). CTR = carpal tunnel release; WALANT = wide awake local anesthesia with no tourniquet; MAC $=$ monitored anesthesia care.

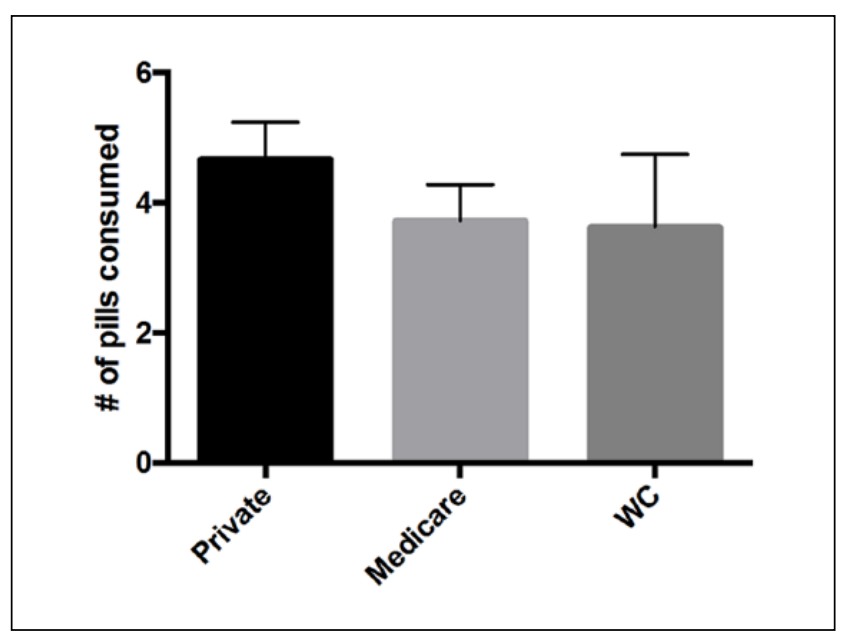

Figure 2. Analysis of opioid consumption based on insurance type.

Note. There was no difference in average opioid consumption when we compared insurance type: Patients with private insurance consumed 4.67 pills compared with 3.72 pills for Medicare patients and 3.62 pills for worker's compensation patients (WC) $(P=.47)$.

significant difference in average opioid consumption $(P=$ $.24)$.

\section{Analysis of Opioid Consumption Based on Opioid Prescribed}

Types of opioid prescribed included Percocet ${ }^{\mathrm{TM}}$ or oxycodone equivalent, Vicodin ${ }^{\mathrm{TM}}$ or hydrocodone equivalent, and Tylenol 3, and no statistically significant difference in average opioid consumption was identified between these opioid types upon comparison $(P=.85$, Figure 3$)$.

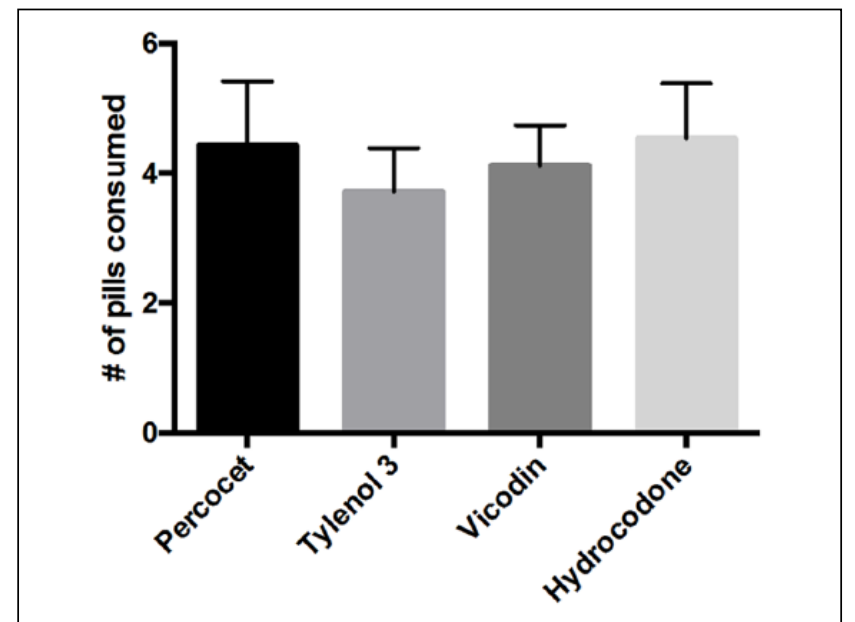

Figure 3. Analysis of opioid consumption based on opioid prescribed.

Note. No statistically significant difference in average opioid consumption was identified between patients who consume Percocet (4.4) versus Tylenol 3 (4.I) versus Vicodin (3.9) versus Hydrocodone (4.6) $(P=.85)$.

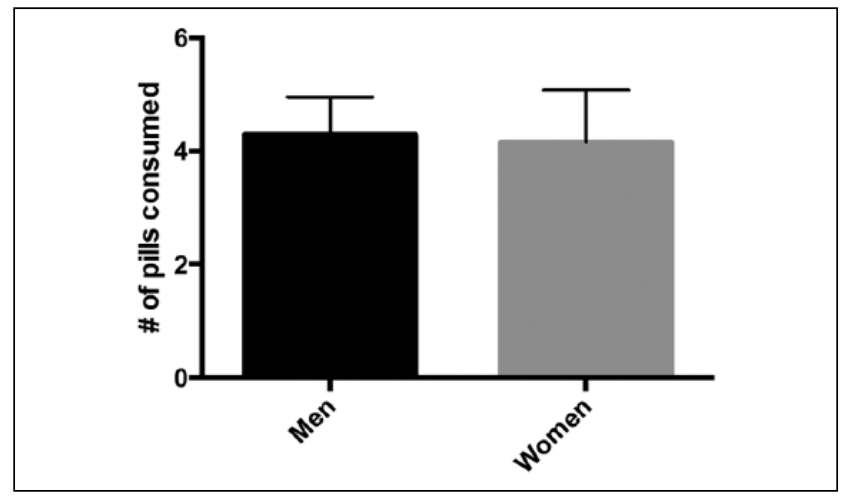

Figure 4. Analysis of opioid consumption based on gender. Note. There was also no statistical difference when we compared the average number of pills consumed by women versus men (4.2 vs 4.3 , $P=.86$; Figure 4).

\section{Analysis of Opioid Consumption Based on Gender}

There was also no statistical difference when we compared the average number of pills consumed by women versus men (4.2 vs $4.3, P=.86$, Figure 4$)$; however, men were more likely to take zero pills (47\%) compared with women $(36 \%)(P \leq .05)$.

\section{Analysis of Opioid Consumption Based on Age}

When we compared opioid consumption with regard to the age of the patients, we found that older patients took significantly fewer pills compared with younger patients $(P<.02$; Figure 5). On average, patients aged between 20 and 39 years took 8.13 pills, patients aged 40 to 59 took 4.89 pills, patients aged 60 to 79 took 3.97 pills, and patients older than 80 took only 2.45 on average. 


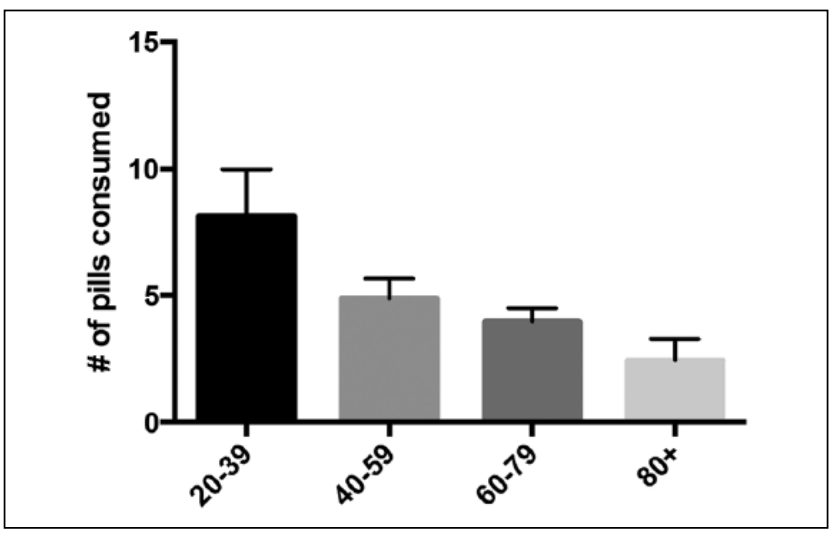

Figure 5. Analysis of opioid consumption based on age. Note. On average, patients aged between 20 and 39 years took 8.13 pills, patients aged 40 to 59 took 4.89 pills, patients aged 60 to 79 took 3.97 pills, and patients older than 80 took only $2.45(P<.02)$.

\section{Discussion}

Because of the growing opioid abuse problem, a more accurate understanding of realistic opioid consumption following surgery is imperative to treat pain appropriately relative to the surgery being performed without leading to excess opioid distribution into society.

Ultimately, we found that many more opioids were prescribed than actually needed. On average, they were overprescribed at a ratio of $5: 1$. The average number of pills consumed was 4.3 , with $87 \%$ consuming 10 or fewer pills, and $41 \%$ of patients did not consume any opioid at all. There were 4478 unused leftover narcotic opioid pills in just this study cohort alone. We found that opioid consumption following CTR is more influenced by age and gender, and less influenced by anesthesia type (WALANT vs MAC), insurance type, or narcotic prescribed. We found that younger patients are significantly more likely to require more opioid analgesia postoperatively.

After performing a post hoc power analysis to determine whether the study was sufficiently powered to detect a difference between opioid consumption in the WALANT versus MAC comparison, we found that in fact the comparison was powered to $83.4 \%$, which represents a relatively wellpowered study.

This study highlights the prevalence of inadvertent overprescription of opioids and will hopefully help to guide prescribing practices in the future. The unintentional overprescribing of opioid postoperatively may be contributing to the current opioid abuse epidemic.

Shortcomings of the study include recall bias and the inherent subjective nature of pill consumption reporting by patients. In addition, we did not take into consideration whether the patients were opioid naive or opioid tolerant prior to surgery.

Future studies may include drastically reducing the number of pills prescribed for CTR surgeries and examining the outcome in terms of patient satisfaction and pain control.

\section{Ethical Approval}

This study was approved by our institutional review board.

\section{Statement of Human and Animal Rights}

All procedures followed were in accordance with the ethical standards of the responsible committee on human experimentation (institutional and national) and with the Helsinki Declaration of 1975, as revised in 2008 .

\section{Statement of Informed Consent}

Informed consent was obtained from all patients for being included in the study.

\section{Declaration of Conflicting Interests}

The authors declared no potential conflicts of interest with respect to the research, authorship, and/or publication of this article.

\section{Funding}

The authors received no financial support for the research, authorship, and/or publication of this article.

\section{References}

1. Alam A, Gomes T, Zheng H, Mamdani MM, Juurlink DN, Bell $\mathrm{CM}$. Long term analgesic use after low risk surgery: a retrospective cohort study. Arch Intern Med. 2012;172:425-430.

2. Cai R, Crane E, Poneleit K, Paulozzi L. Emergency department visits involving nonmedical use of selected prescription drugs-United States, 2004-2008. Morb Mortal Wkly Rep. 2010;59:705-709.

3. Carroll I, Barkelka P, Wang CK, et al. A pilot cohort study of the determinants of longitudinal opioid use after surgery. Anesth Analg. 2012;115:694-702.

4. Chen L, Hedegaard H, Warner M. QuickStats: Number of Deaths From Poisoning, Drug Poisoning, and Drug Poisoning Involving Opioid Analgesics-United States, 1999-2010. Atlanta, GA: Centers for Disease Control and Prevention; 2013.

5. Governale L. Outpatient prescription opioid utilization in the U.S., years 2000-2009. Presentation for US Food and Drug Administration; July 22, 2010; Silver Spring, MD http:// www.fda.gov/downloads/AdvisoryCommittees/Committees MeetingMaterials/Drugs/Anesthetic AndLifeSupport DrugsAdvisoryCommittee/UCM220950.pdf.

6. Manchikanti L, Helm S, Fellows B, et al. Opioid epidemic in the United States. Pain Physician. 2012;15:ES9-ES38.

7. Paulozzi L, Jones CM, Mack KA, Rudd RA. Centers for Disease Control and Prevention. Vital signs: overdoses of prescription opioid pain relievers_-United States, 1999-2008. Morb Mortal Wkly Rep. 2011;60:1487-1492.

8. Rodgers J, Cunningham K, Fitzgerald K, Finnerty E. Opioid consumption following outpatient upper extremity surgery. J Hand Surg Am. 2012;37:645-650.

9. Substance Abuse and Mental Health Services Administration. Results From the 2011 National Survey on Drug Use and Health: Summary of National Findings. NSDUH Series H-44, HHS Publication No. (SMA) 12-4713. Rockville, MD: Substance Abuse and Mental Health Services Administration; 2012.

10. Volkow ND, McLellan TA, Cotto JH. Characteristics of opioid prescriptions in 2009. JAMA. 2011;305:1299-1301. 